\title{
Cognitive and physical perceived stress traits associated with lactose intolerance displayed within the Black, Asian and minority ethnic UK population
}

\author{
E. Isoa ${ }^{1}$ and S. Sarkar ${ }^{1}$ \\ ${ }^{1}$ Department of Sports and Rehabilitation, Bournemouth University, Bournemouth, UK
}

Lactose intolerance (LI) has vastly soared within the global population in recent years, accounting for up to $75 \%$ of the overall population globally ${ }^{(1)}$. In the United Kingdom (UK), dairy consumption has been an essential requirement in meeting calcium and vitamin D levels. However, ethnic minorities described as 'BAME' inclusive of Black, Asian, and Minority Ethnic populations struggle to ingest or utilise dairy, leading to $\mathrm{LI}^{(2)}$. In addition, LI has been postulated to cause several symptoms, including headaches, 'brain fog' and fatigue alongside typical gastrointestinal (GI) symptoms of bloating and diarrhoea (5\% UK). Moreover, stress can play a factor in food intolerance. Therefore, this study aimed at the relationship between LI and perceived stress. The study aims to explore the relationship of LI within the BAME UK population via perceived stress.

The total sample of $n=317$ people were recruited by simple random sampling from social media and professional networks including Twitter, LinkedIn, Facebook, Snapchat, YouTube, TikTok and WhatsApp, shared via Jisc Online Surveys@ for data collection. Statistical tests included correlation, independent $t$-test and ANOVA, which was created and analysed via Statistical Package for the Social Sciences ${ }^{\circledR}$ (Chicago, IL) by International Business Machines (IBM) version 27.

People of the BAME population who took part in the study included, Black British - African \& Caribbean (246), Asian British Indian, Pakistani, Bangladeshi \& Chinese (36), Mixed/Multiple ethnic groups (20) and other ethnic groups (15).

Rating scores were based on a 5-point Likert Scale questionnaire within the present study, aligned with perceived stress (cognitive, emotional, behavioural and physical). This was influenced by Cohen's (1983) perceived stress scale (PSS) model. Thus, phrases such as cognitive, emotional, behavioural and physical, collectively relate to stress, indicating rationale of word choices as usage of measurement (PSS scores).

The current study's findings revealed that a weak positive correlation was identified $(r=.472, p \leq .001, M=10.63, S D=3.60$ [cognitive], $M=9.04, S D=3.61$ [physical]). In addition, the Black population were the most stressed with LI. The Asian population also outlined signs of stress with LI (no significance - compared to the Black population); two of the largest ethnic minorities in the UK Females were more stressed than males influenced by LI.

The present study outlined a relationship between LI and perceived stress in the BAME UK population. This brought the attention that psychological and physiological stress alongside LI needs further investigation where correlations should not be solely reliant. Therefore, understanding the susceptibility of LI and perceived stress within this population needs to be the next step moving forward. Furthermore, to comprehend the phenomena as to why this demographic is predominantly affected by LI.

\section{Acknowledgments}

First and foremost, I would like to thank my supervisor, Dr Swarjit Sarkar, for his high enthusiasm and faith in me to complete my dissertation, inspiring me to do well during this masters course. His level of expertise has been second-to-none, especially with the theme of ethnic minorities in nutrition, contributing to this project. In addition, he has been a great mentor. Thank you, Swarjit! I would like to acknowledge my girlfriend Elizabeth Fabiyi, who has been my rock and strength, motivating me to reach the best of my abilities, full of positivity. When I had selfdoubt, she always picked me up, even if it is tough love. Thank you, my dear! Finally, I would like to thank my mum, Henrietta Telkman, for being an inspiration and role model in my life, education, and best friend! Love you, mum! Most importantly, I want to thank the fantastic people who took part in my study through my data collection. The exposure has been phenomenal alongside the completed questionnaire responses. Thank you so much!

\section{References}

1. Micic D, Rao VL \& Rubin DT (2019) JAMA. 322(21), 2138.

2. Goh LH, Goh KL \& Said RM (2018) J. Gastroenterol 2(6), 307-310. 